\title{
Coronavirus \& the Heart - Acute Heart Attack and Coronary Artery Disease - Pathophysiology
}

\author{
Tanay Subramanian ${ }^{1}$ and James Patel ${ }^{1}$
}

${ }^{1}$ Dougherty Valley High School, San Ramon, CA, USA

\section{ABSTRACT}

The coronavirus has consumed over 648,000 lives and 16.2 million people worldwide have tested positive for this virus, making it a pandemic, as of July 26, 2020. There are 4.2 million cases of COVID-19 in the United States and 146,000 deaths, with the number of cases rising by thousands per day (COVID-19 Map, 2020). This makes it difficult to pinpoint an accurate number for coronavirus research as it is ever-changing. The primary symptoms of the virus include severe pneumonia, dry cough, and fatigue, although patients may be asymptomatic as well, making it harder to diagnose. Research reveals the unfortunate reality of yet another symptom associated with the virus - myocardial injuries. There seems to be a significant correlation between the coronavirus and cardiac damages, which in turn leads to acute heart problems. This raises a flag to many coronavirus patients - the possible link between COVID-19 and cardiovascular diseases such as acute heart failure and myocardial infarctions.

Research from the Journal of the American Medical Association (JAMA) has revealed that $35.3 \%$ of tested patients had underlying cardiovascular disease including hypertension, coronary heart disease, and cardiomyopathy and 27.8\% exhibited myocardial injury indicated by elevated levels of molecules called troponin (Guo et al., 2020).

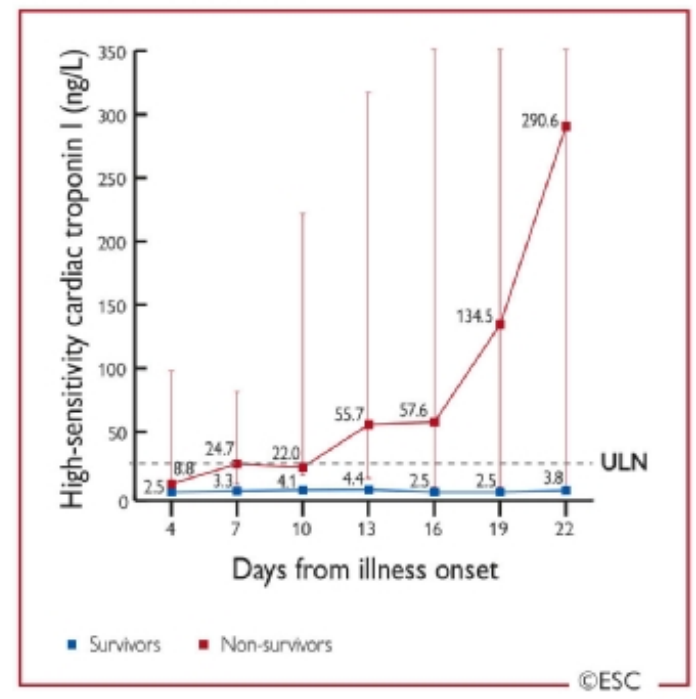

Figure 1. Temporal changes in cardiac troponin I concentrations from illness onset in hospitalized COVID-19 patients, between survivors and non-survivors. 
Troponin is a complex of troponin $\mathrm{C}(\mathrm{TnC})$, troponin $\mathrm{T}(\mathrm{TnT})$, and troponin $\mathrm{I}(\mathrm{TnI})$, which are all regulatory proteins. Cardiac troponins are a class of proteins found in muscle fibers of the heart, to which calcium ions bind in order to regulate the muscular contractions of the heart (Troponin - an Overview | ScienceDirect Topics, n.d.). If the concentration of troponins is significantly higher than the optimum amounts, myocardial injuries such as heart failure and heart attack are evident. In the United States, troponin I is most commonly measured for diagnosis and this is a specific fraction of troponin which prevents myosin from binding to actin to relax muscles. Studies find that the coronavirus interferes with troponin concentration, causing these regulatory proteins to accumulate rapidly. But what exactly from this virus causes a surge in troponin levels?

Troponin elevations are due to the inflammation of the lungs associated with pneumonia, a key symptom of the coronavirus, which then sends an immune response to other organs, including the heart's muscles, causing them to inflame. This inflammation of the heart muscles known as myocarditis is associated with viral infections like COVID-19, as well as influenza (RWJBarnabas Health, Inc., n.d.). Additionally, myocarditis can cause surrounding arteries to develop clots which can lead to hypertension, heart attack, and ultimately result in cardiac necrosis, the death of heart cells, which can be fatal. When these heart cells become damaged from the obstruction of the heart vessels, they release troponin I into the bloodstream, causing troponin levels to surge. Optimal levels of troponin I are below $0.04 \mathrm{ng} / \mathrm{ml}$. Levels significantly greater than $0.04 \mathrm{ng} / \mathrm{ml}$ and above $0.40 \mathrm{ng} / \mathrm{ml}$ causes the muscles to become less relaxed and more rigid, creating a sudden blockage of blood flow to the heart, resulting in acute coronary syndrome (Villines, 2019).

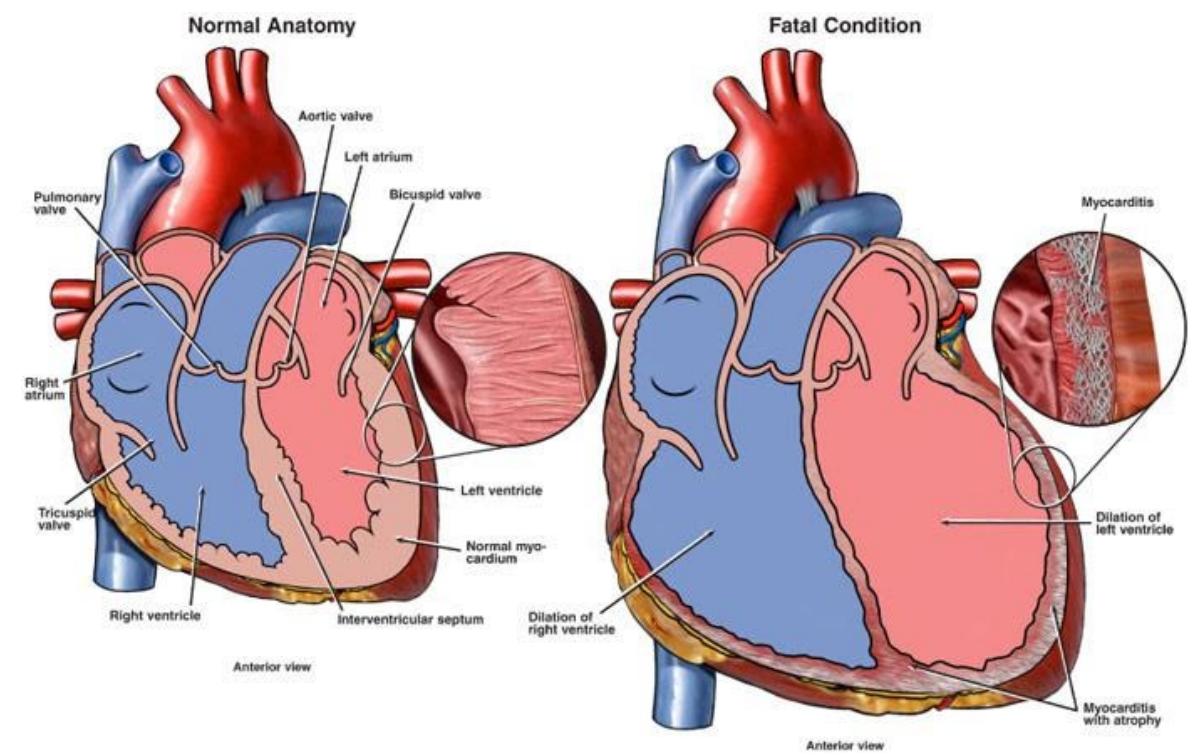

Figure 2. Differences in the myocardium between a normal heart and a heart affected with myocarditis.

According to mortality data released by the National Health Commission of China, 35 percent of the patients who died from COVID-19 had a history of high blood pressure and 17 percent had a history of coronary heart disease (Kayat, 2020). COVID-19 can also cause viral Vasculitis, the inflammation of the coronary arteries due to the immune system's damaging of blood vessels, which causes an acute blockage that leads to the death of myocardial cells and angina (Malas \& Obeid, n.d.). These cardiac injuries release troponins I and T into the blood which proves an acute 
heart attack (ESC Guidance for the Diagnosis and Management of CV Disease During the COVID-19 Pandemic, 2020). In addition to myocardial infarctions, Vasculitis can also contribute to hypertension and aortic aneurysm.

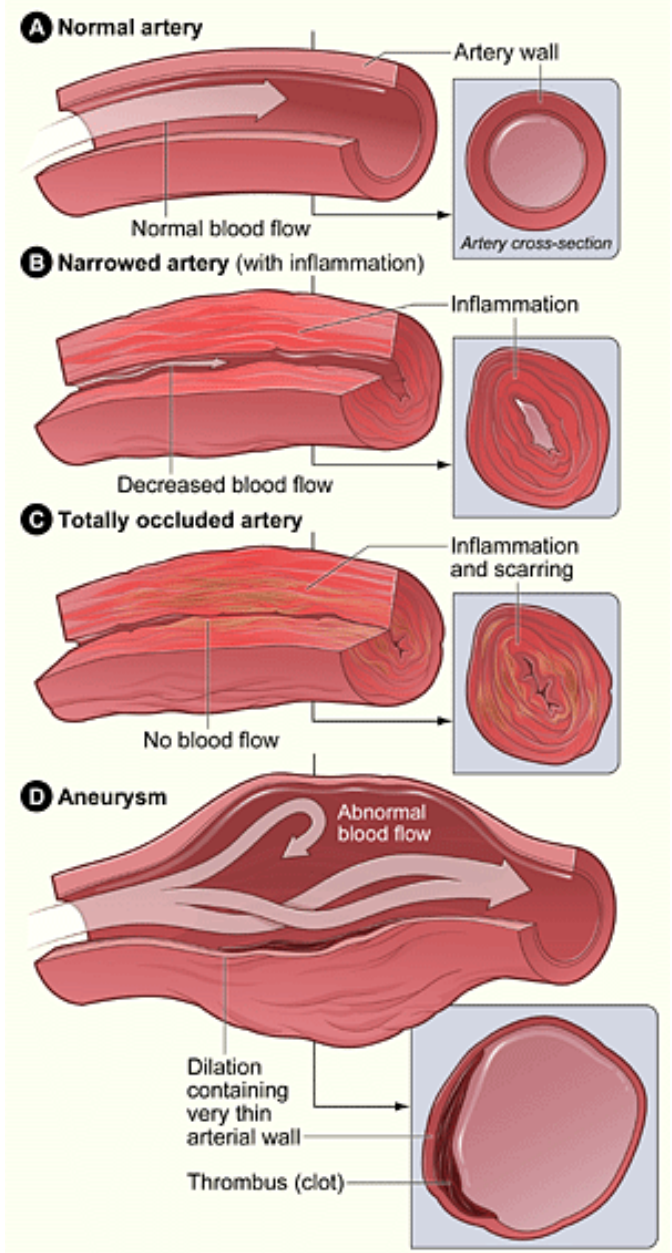

Figure 3. Differences in blood flow between normal arteries and obstructed and inflamed arteries.

Inflammation occurs as the body's defense mechanism against pathogens but can result in the inflammation of other organs as well. The defense system initiated by the coronavirus results in multi-organ failure because the virus causes cardiovascular disease, acute respiratory distress syndrome (ARDS), kidney failure, and malfunctioning of the central nervous system to limit smell and taste upon early infection. The most recent finding is that the disease runs a milder course if an affected patient has a loss of sense of smell or taste.

Once the inflammation reaches the heart, the virus can proliferate within the myocardium and cause a cascade of inflammatory responses that ultimately disrupts the electrical rhythms of the heart, leading to heart failure. Congestive heart failure often progresses into pulmonary edema because the heart is not able to pump blood to the body efficiently, so blood returns to the veins that transport blood through the lungs. As the pressure in these veins increases from the accumulation of blood, hypertension occurs, so fluids are pushed into the alveoli of the lungs and the buildup of fluids causes difficulty in breathing (Pulmonary Edema, 2020). 
Italian autopsies prove a vast spread of vascular blockades like disseminated intravascular clotting (DIC), which causes multi-organ failure and increases the likelihood of comorbidities. A recent CDC report reveals that $90 \%$ of hospitalized coronavirus patients have comorbidities such as hypertension, obesity, and diabetes (Zaiets \& Padilla, 2020). DIC requires parenteral anticoagulants especially in acutely ill hospitalized or ICU patients on the ventilators. Once on the ventilator, it becomes a challenge to get off of it, as $\mathrm{SpO} 2$ (oxygen saturation) remains in the low $80 \%$, causing hypoxemia which can be fatal. Also, shortness of breath, a major symptom of COVID-19, can result if SpO2 saturation is less than $90 \%$. However, research shows that the antiviral drug Remdesivir improves $\mathrm{SpO} 2$ to more than 90\%. UC Davis Health reports that in a clinical trial which prescribed Remdesivir to coronavirus patients, $68 \%$ of patients had improved oxygen level as indicated by increased $\mathrm{SpO} 2$ levels, more than half of patients on ventilators were taken off the device, and after a month, $84 \%$ of patients had been discharged (UC Davis Health, 2020). This is promising as it shows that hospitalized but not severely ill patients could be prescribed antiviral medications intravenously to halt coronavirus's progression. Alternatively, some patients could be considered for Direct Oral Anticoagulants (DOAC) to prevent them from progressing to DIC.

OVERALL, ONE OR MORE UNDERLYING CONDITION

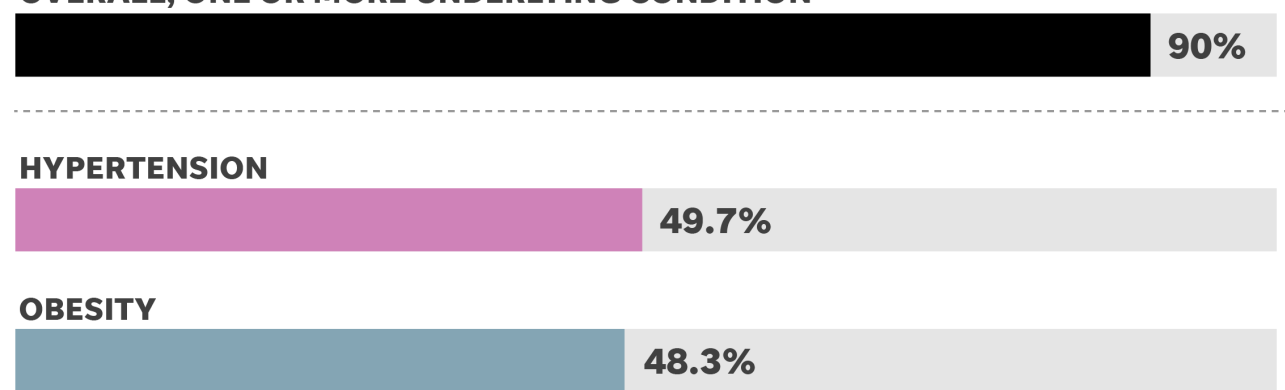

CHRONIC LUNG DISEASE

\section{$34.6 \%$}

DIABETES

$28.3 \%$

CARDIOVASCULAR DISEASE

$27.8 \%$

Figure 4. Leading conditions involved in comorbidities of coronavirus patients.

Creatine kinase-Muscle and Brain (CK-MB) is another protein that is prevalent in heart muscle cells. Similar to Troponin I, CK-MB is released from blood cells in response to cardiac damage from DIC and viral Vasculitis which inflames the coronary arteries. Widespread Vasculitis causes intravascular clotting which causes multi-organ failure since deep vein thrombosis (DVT) can block an essential vein that provides blood to various organs. Intravascular clotting or thrombus can be detected by the D-Dimer (a protein present in the blood after clotting) blood test, which can also be used to detect DVT and pulmonary embolism. Hence, besides IV antiviral Remdesivir medication, 
Decadron, Heparin, and Low Molecular Weight Heparins (LMWH) like Lovenox, all of which should be injected intravenously, can be used to combat the inflammatory and clotting symptoms (Renneberg \& Glatz, n.d.).

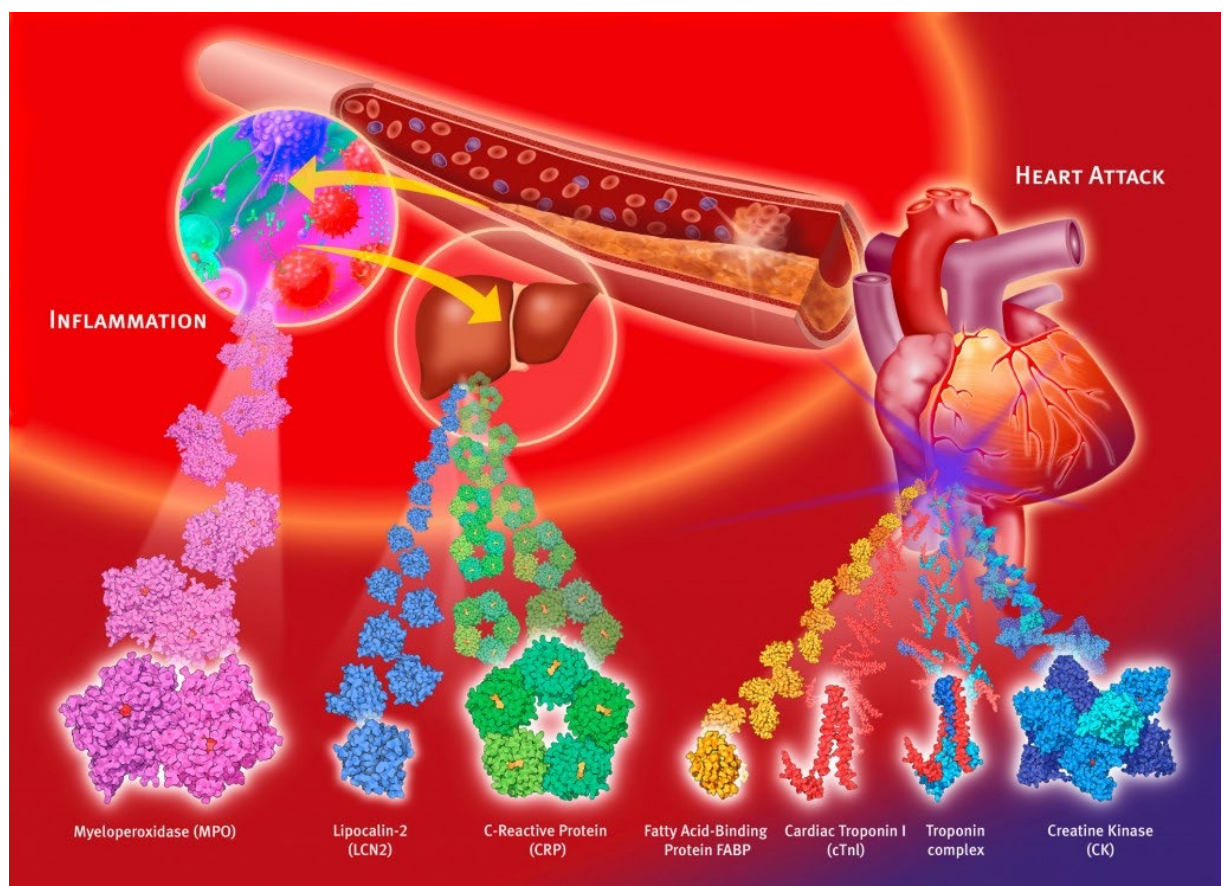

Figure 5. The effect of inflammation on heart attack and the release of proteins into the bloodstream.

Along with CK-MB being released in response to increased pressure on the myocardium due to heart failure and AMI from viral Vasculitis following exposure to COVID-19, cardiac myocytes release N-terminal prohormone BNP (NTproBNP), an inactive hormone precursor (Iqbal et al., 2012). An NT-proBNP test can be conducted to diagnose congestive heart failure which occurs post acute heart attack. These prohormone levels increase when cardiac problems are present. Healthy levels of NT-proBNP should be less than $125 \mathrm{pg} / \mathrm{ml}$ for patients aged 0-74 years and less than $450 \mathrm{pg} / \mathrm{ml}$ for patients aged 75-99 years. Quantities greater than $450 \mathrm{pg} / \mathrm{ml}$ for patients under age 50 and greater than $900 \mathrm{pg} / \mathrm{ml}$ for patients aged 75-99 years indicate heart failure (NT-ProB-Type Natriuretic Peptide (BNP), 2019).

Cytokines are crucial proteins of the immune system. While they are helpful in moderate amounts, severe inflammation detected by Interleukin 6 (IL-6) can cause a surge in cytokine levels resulting in a cytokine storm, where the body attacks its cells and tissues, instead of fighting the virus (Goodman, 2020). Fortunately, the cytokine storm can be treated with monoclonal antibodies (mAB) which mimics the immune system's defense to attack the virus.

There seems to be a positive correlation between age and susceptibility to the disease. The CDC reports that $80 \%$ of COVID-19 deaths reported in the U.S. have been in adults older than 65 years old (McCarthy, 2020) because their immune systems weaken, making them vulnerable to diseases. Similarly, the low socioeconomic class is more inclined to contract the virus as they lack access to quality health care and live in unsanitary, crowded conditions where transmission is more likely. Furthermore, workers with lower levels of education are less likely to work from home, so they are less able to physically distance than those with higher levels of education (Finch \& Finch, 2020). Hypertensive patients are also at a greater risk of getting infected since the immune response is amplified with a higher blood 
pressure. A stronger immune response will only increase inflammation, escalating DIC, which can result in a myocardial infarction or even an ischemic stroke. Angiotensin receptor blockers (ARBs) and non-dihydropyridine calcium antagonists (NDCAs), which function to lower blood pressure by dilating arteries, can be given to hypertensive COVID-19 patients to maintain blood pressure, but not to combat the virus itself.

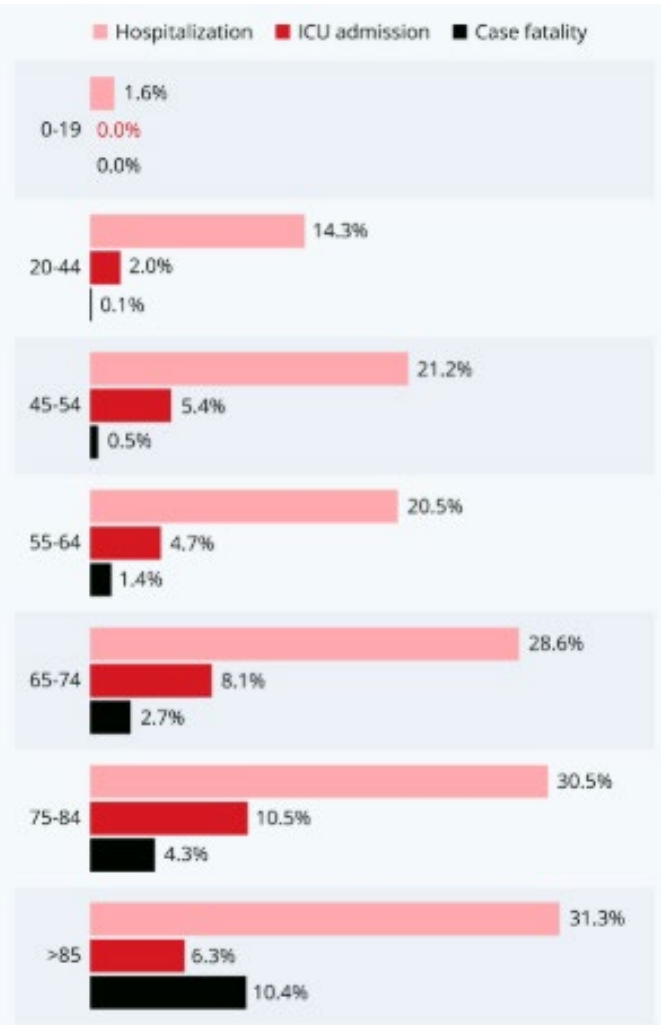

Figure 6. COVID-19 correlation with different age groups, factoring hospitalization, ICU admission, and fatality rates.

As far as preventive measures are concerned, little can be done to entirely prevent the development of cardiovascular disease in coronavirus patients. However, medications such as Diltiazem, Verapamil, and Metoprolol can help reduce the likelihood of cardiovascular disease and high blood pressure. Diltiazem and Verapamil work as calcium channel blockers which can be used to treat arrhythmia and hypertension, among other heart irregularities because less calcium will bind to troponin I, so the cardiac muscles become relaxed. Metoprolol is a beta channel blocker which is more commonly used nowadays. This medication blocks epinephrine from binding to its hormone receptors and promotes vasodilation to improve blood flow and lower blood pressure. Both calcium and beta channel blockers decrease the oxygen demand of the heart but beta blockers are proven to be more efficient in decreasing this demand to reduce the stress on the heart.

A research group in Greece found that colchicine, a medicine commonly used to treat gout, improves time to clinical deterioration, and is especially beneficial among patients with cardiovascular disease. In their study, 10-day survival was $97 \%$ in the colchicine group versus $83 \%$ among controls, showing statistical significance. This, along with its anti-inflammatory and anti-thrombogenic effects suggest that colchicine might combine cardiovascular safety 
with a favorable overall risk-benefit profile, which could reduce the myocardial injuries associated with COVID-19 (Deftereos, 2020).

Another encouraging therapy is giving convalescent plasma, containing the Immunoglobulin G (IgG) antibody, to halt the progression of COVID-19 to the acutely ill patients that require ventilators in the ICU (Valk et al., 2020). However, it is difficult to obtain this since few willing patients are fully recovered and discharged. Moreover, we would need enough blood transfusions for over 648,000 people.

Unfortunately, a vast population is in support of the medicine hydroxychloroquine being released to the general public. Hydroxychloroquine is effectively used to treat malaria, rheumatoid arthritis, and lupus, but is now believed to also treat COVID-19 in non-hospitalized patients. However, this drug actually causes cardiac complications because it precipitates prolonged QT syndrome (fast and chaotic heartbeats) which can result in lethal cardiac arrhythmia like ventricular tachycardia that can degenerate into ventricular fibrillation. This causes death unless a cardioverter defibrillator is promptly used to countershock the heart. Therefore, hydroxychloroquine should only be used in hospitalized patients under cardiac monitoring so if any arrhythmia ensues, the condition can be addressed immediately.

Novel studies suggest that Methotrexate, a powerful anti-inflammatory drug used to treat rheumatoid arthritis, may be successful in fighting the autoimmune diseases initiated by COVID-19. This drug works by decreasing monocyte (white blood cell) proliferation to produce a less significant immune response. Therefore, the inflammation isn't ubiquitous in the body and can prevent multi-organ failure. However, before further implementation and release to the public, Methotrexate must be tested in clinical trials to measure its success and potential side effects (Safavi \& Nath, 2020).

A healthy diet including probiotics and zinc may be effective to combat the coronavirus. Clinical evidence shows that certain probiotic strains and cofactors help to prevent bacterial and viral infections like sepsis and respiratory tract infections.

\section{Conclusion}

My research supports that the coronavirus can cause acute myocardial injuries from inflammation which affects various organs. The pathophysiology of the coronavirus causes cardiac injuries through viral Vasculitis, which releases troponins into the bloodstream to narrow arteries and reduce blood flow, leading to heart and respiratory illnesses.

Widespread antigen RNA testing is crucial since positive cases and their contacts, even if asymptomatic, can be self-quarantined for a couple of weeks as this is an incumbent measure to curb the pandemic. Equally important is wearing a mask outside of your house as the tiny particles from coughing and sneezing can be carried far away by the wind. We need to consider each asymptomatic person as a potential carrier of COVID-19 and the mask will help others as well as ourselves from contracting the disease. Some cities in Texas and California have enforced stricter regulations for wearing masks, by issuing fines to nonconformists. The 6 feet distancing enforced is ineffective because it's not scientifically proven as absolute safe social distancing. Research at MIT and other leading institutions have proven that 27 feet is an entirely safe distance, which is mostly impractical to maintain. Therefore, wearing a mask over the nose and mouth is the only proven measure that can help us all in containing the pandemic (Lotan, 2020). 


\section{References}

COVID-19 Map. (2020, July 26). Johns Hopkins Coronavirus Resource Center. https://coronavirus.jhu.edu/map.html

Deftereos, S. (2020, June 24). Effect of Colchicine vs Standard Care on Cardiac and Inflammatory Biomarkers and Clinical Outcomes in Patients Hospitalized With Coronavirus Disease 2019: The GRECCO-19 Randomized Clinical Trial. Infectious Diseases | JAMA Network Open | JAMA Network. https://jamanetwork.com/journals/jamanetworkopen/fullarticle/2767593

ESC Guidance for the Diagnosis and Management of CV Disease During the COVID-19 Pandemic. (2020, June 10). European Society of Cardiology. https://www.escardio.org/Education/COVID-19-and-Cardiology/ESC-COVID-19Guidance

Finch, W., \& Finch, M. (2020, June 15). Poverty and Covid-19: Rates of Incidence and Deaths in the United States During the First 10 Weeks of the Pandemic. Frontiers. https://www.frontiersin.org/articles/10.3389/fsoc.2020.00047/full

Goodman, B. (2020, April 17). Cytokine Storms May Be Fueling Some COVID Deaths. WebMD. https://www.webmd.com/lung/news/20200417/cytokine-storms-may-be-fueling-some-covid-deaths

Guo, T., Fan, Y., Chen, M., Wu, X., Zhang, L., \& He, T., et al. (2020, March 27). Cardiovascular Implications of Fatal Outcomes of Patients With Coronavirus Disease 2019 (COVID-19). Cardiology | JAMA Cardiology | JAMA Network. https://jamanetwork.com/journals/jamacardiology/fullarticle/2763845

Iqbal, N., Wentworth, B., Choudhary, R., Landa, A., Kipper, B., \& Fard, A., et al. (2012, June). Cardiac biomarkers: New tools for heart failure management. Cardiovascular Diagnosis \& Therapy. http://cdt.amegroups.com/article/view/685/754

Kayat, S. (2020, April 16). Doctor's Note: Coronavirus and Heart Disease. Coronavirus Pandemic | Al Jazeera. https://www.aljazeera.com/indepth/features/doctor-note-coronavirus- heart-disease -200416072036397.html

Lotan, G. (2020, March 31). Six-foot rule to protect against coronavirus may not be enough, MIT professor says. Boston Globe. https://www.bostonglobe.com/2020/03/31/nation/six-foot-rule-protect-against-coronavirus-is-questionable-mit-professor-says/

Malas, M., \& Obeid, T. (n.d.). Vasculitis | Society for Vascular Surgery. Society for Vascular Surgery. https://vascular.org/patient-resources/vascular-conditions/vasculitis 
McCarthy, N. (2020, March 19). How COVID-19 Affects Different U.S. Age Groups. Statista Infographics. https://www.statista.com/chart/21173/hospitalization-icu-admission-and-fatality-rates-for-reported-coronavirus$\underline{\text { cases/ }}$

NT-proB-type Natriuretic Peptide (BNP). (2019, April 25). Cleveland Clinic. https://my.clevelandclinic.org/health/diagnostics/16814-nt-prob-type-natriuretic-peptide-bnp

Pulmonary Edema. (2020, July 2). MedlinePlus U.S. National Library of Medicine. https://medlineplus.gov/ency/article/000140.htm

Renneberg, R., \& Glatz, J. (n.d.). The Earliest Plasma Marker for Myocardial Infarction. Lab\&more. http://www.int.laborundmore.com/archive/852368/The-earliest-plasma-marker -for-myocardial-infarction.html

RWJBarnabas Health, Inc. (n.d.). RWJBarnabas Health. https://www.rwjbh.org/treatment-care/heart-and-vascular$\underline{\text { care/diseases-conditions/myocarditis/ }}$

Safavi, F., \& Nath, A. (2020, August 15). Silencing of Immune Activation with Methotrexate in Patients with COVID-19. PubMed Central (PMC). https://www.ncbi.nlm.nih.gov/pmc/articles/PMC7247505/

Troponin - an overview | ScienceDirect Topics. (n.d.). ScienceDirect. Retrieved July 18, 2020, from https://www.sciencedirect.com/topics/veterinary-science-and-veterinary-medicine/troponin \#:\%7E:text=Troponin $\% 20$ is $\% 20 \mathrm{a} \% 20$ complex $\% 20$ of $\% 20$ three $\% 20$ regulatory $\% 20$ proteins $\% 2 \mathrm{C} \% 20$ troponin $\% 20 \mathrm{C}$,produces $\% 20$ conformational $\% 20$ change $\% 20$ in $\% 20 \mathrm{TnI}$

UC Davis Health. (2020, April 16). Remdesivir Improved Majority of Patients Hospitalized With Severe COVID19. https://health.ucdavis.edu/health-news/newsroom/remdesivir-improved- majority-of-patients-hospitalized-with$\underline{\text { severe-covid-19/2020/04 }}$

Valk, S., Piechotta, V., Chai, K., Doree, C., Monsef, I., \& Wood, E., et al. (2020, May 14). Convalescent Plasma or Hyperimmune Immunoglobulin for People with COVID-19: A Rapid Review. PubMed. https://pubmed.ncbi.nlm.nih.gov/32406927/

Villines, Z. (2019, June 7). What is the normal range for troponin levels? MedicalNewsToday. https://www.medicalnewstoday.com/articles/325415\#normal-troponin-range

Zaiets, K., \& Padilla, R. (2020, April 15). Coronavirus, diabetes, obesity and other underlying conditions: Which patients are most at risk? USA Today. https://www.usatoday.com/in-depth/news/2020/04/15/coronavirus-risk-90patients-had-underlying-conditions/2962721001/ 